\title{
UNA EDUCACIÓN PLURILINGÜE PARA EL EJERCICIO DE UNA NUEVA CIUDADANÍA
}

\section{A MULTI-LINGUAL EDUCATION FOR THE EXERCISE OF A NEW CITIZENSHIP}

\author{
Barbara da Silva Santana Lopes ${ }^{1}$ \\ Facultad de Humanidades y Ciencias de la Educación \\ Universidad Nacional de La Plata \\ barbarasslopes@gmail.com
}

\begin{abstract}
Resumen
Este artículo pretende cuestionar las decisiones gubernamentales respecto de la participación de lenguas que circulan en el ambiente universitario y, como consecuencia, en la construcción de saberes, para luego proponer una mirada plurilingüe con todo lo que esta implicaría: un cambio en el paradigma que permitiera la ampliación de la subjetividad en el ejercicio de una nueva ciudadanía. Para llevar a cabo dicha propuesta, remitiremos a los estudios de variación que se apoyan en la esencia pragmática de los eventos comunicativos, así como también nos detendremos en el punto de partida desde el repertorio de hablantes que nos ofrecen los estudios translingüísticos. Puntualmente, expondremos cómo las políticas de integración regional contribuyeron, dentro de sus posibilidades, a la ampliación, por ejemplo, del Departamento de Lenguas y Literaturas Modernas de la Universidad Nacional de La Plata, con la implementación del Profesorado en portugués en 2014 y con ello se consolidó la participación de la lengua portuguesa en eventos científicos en esta Universidad y en la Argentina. ${ }^{2}$
\end{abstract}

Palabras clave: Ciudadanía - Lenguas - Enseñanza - Universidad pública - Derechos 


\begin{abstract}
This article aims to question government's decisions regarding the inclusion of different languages that circulate in the university environment and that have a fundamental role in the academic knowledge construction. Therefore, we propose a multilingual perspective that implies: a change in paradigm that would allow subjectivities expansion in the exercise of a new citizenship. To carry out this proposal, we will refer to the variation studies that are based on the pragmatic essence of communicative events, as well as we will stop at the starting point from the repertoire of speakers that translinguistic studies offer us. Specifically, we will show how regional integration policies contributed, within their possibilities, to the expansion of the Department of Modern Languages and Literatures of the National University of La Plata, leading to the implementation of Portuguese Teaching Staff in 2014 and with this the participation of the Portuguese language in scientific events at this University and in Argentina.
\end{abstract}

Keywords: Citizenship - Languages - Teaching - Public University - Rights 


\section{INTRODUCCIÓN}

Desde el año 1991, las políticas de integración regional y, con ello, la aproximación entre los países del Mercosur incentivó la circulación de lenguas vecinas en contextos comerciales y también educativos. En Brasil, las escuelas empezaron a ofrecer el español como lengua optativa desde 2005 y en la Argentina se legisló en 2009 la oferta del portugués brasileño como disciplina curricular.

En el marco de las decisiones que ampliarían el vínculo entre los países, se incentivó la reflexión acerca de una nueva política lingüística establecida en la creación del Mercosur (Arnoux, 2010). En este libro, la autora se expresa acerca de la posibilidad de una identidad colectiva que abarque los países y las lenguas del Mercosur:

Aprender otra lengua es introducirse progresivamente en esos juegos (juegos de resonancias culturales al que cada lengua está asociada) que van modelando la subjetividad en tensión y acuerdo con la lengua y la cultura propias. Esta dinámica multiplicada en el espacio sudamericano hace posible pensar en la conformación de un imaginario colectivo que sostenga el ejercicio de una nueva ciudadanía. (Arnoux, 2010, p.20)

Esta identidad colectiva estaría posibilitada por las políticas públicas locales y la geopolítica comprometida con la ampliación de los derechos ciudadanos, pero también -y sobre todo- con el ensanchamiento de la propia identidad. Se trata de una integración no solo de la lengua y de su economía (Bourdieu, 2008), sino también de su cosmovisión. Es decir, un cambio que afecte la subjetividad de la población. La posibilidad de dicha mudanza identitaria se vería fortalecida por el proceso de globalización (Arnoux, 2010) que de a poco, atendiendo a la demanda mundial, desestabilizaría las lenguas nacionales en tanto amplía su participación en espacios "ajenos".

Sin embargo, es importante no dejar pasar que si, por un lado, la globalización puede desestabilizar las lenguas nacionales, por otro lado, es importante observar cuáles son las lenguas elegidas para ocupar espacios ajenos y si el proceso de globalización no termina por marginalizar aún más las lenguas minoritarias. Particularmente, interesa este último aspecto, si tenemos en cuenta los cambios producidos en los últimos años respecto de las políticas lingüísticas llevadas adelante por los distintos gobiernos. En este sentido, Arnoux (2019) sostiene:

El neoliberalismo parece desdeñar el valor identitario de las lenguas en beneficio del valor comercial o busca sacar beneficios económicos de la identidad. Por supuesto que esto varía según la situación -central, secundaria o periférica- de los países y en el caso de los más poderosos ambos valores conviven. Estados Unidos, por ejemplo, si bien insiste en el valor nacional del inglés en el interior de sus fronteras, no puede dejar de considerar el español que, aunque subalternizado, constituye un mercado importante. Además, es consciente de que un inglés global, aunque sea una variedad desprendida de los rasgos nacionales en la 
medida en que debe simplificarse, lo beneficia económicamente ya que constituye no solo una fuente de negocios relacionados con la venta de productos pedagógicos y lingüísticos, sino que también activa el consumo de otros productos. (Arnoux, 2019, p.27)

Nos interesa interrogarnos acerca de la presencia -y la ausencia- de lenguas en el sistema universitario, así como los distintos formatos autorizados para generar conocimiento. Las políticas de integración regional entre los países del Mercosur y de América Latina visibilizaron una problemática lingüística vinculada a la identidad regional: ¿cuáles son las lenguas, los idiomas nacionales, que ponen en funcionamiento los sistemas institucionales? ¿Cómo se espera que la Universidad pública sea integradora si restringe la producción y transmisión de conocimientos a un puñado de lenguas ignorando otras que constituyen la realidad lingüística de la población a la que dirige sus esfuerzos?

Atendiendo a esas problemáticas, en este trabajo proponemos un diálogo entre dos teorías lingüísticas de base sociolingüística, para mostrar que es posible ampliar las categorías que caracterizan la lengua y también valorar el repertorio de cada hablante en contexto. Nos referimos a los estudios de variación lingüística, que nos permiten advertir y explicar, a través del análisis, sesgos culturales que modelan las gramáticas (Martínez, $2015,2018,2020$ ) y la perspectiva Ilamada translanguaging (Otheguy et al., 2015) que valora la identidad lingüística de seres con múltiples y diferenciados rasgos identitarios y con un bagaje de recursos propios en la construcción de significados.

Creemos que dichas perspectivas pueden contribuir a producir conocimiento de base sobre el funcionamiento de los lenguajes y sobre las estrategias de hablantes bilingües e impactar en una propuesta de universidad integradora, plurilingüe, independiente, común y acorde a las necesidades locales, capaz de participar activamente -desde sus aportes- en las relaciones exteriores.

\section{El contexto político}

Se espera que la Universidad pública ofrezca la posibilidad de que la ciudadanía pueda ejercer sus derechos como sujetos sociales: el derecho a la educación que les permita participar en la producción de saberes. Sin embargo, sabemos que la realidad ha sido históricamente otra, que el ambiente universitario ha tenido un sesgo fuertemente elitista, y como resultado ha revelado las violencias simbólicas -y otras no tan simbólicasque han reforzado la estigmatización de ciertos sectores sociales (Gentili, 2008).

Sin embargo, las políticas de integración regional mencionadas más arriba tuvieron su correlato en el desarrollo de políticas educativas tendientes a la ampliación del acceso a la educación superior de otros sectores sociales, lo que generó modificaciones en la composición del estudiantado, si bien no resolvió las desigualdades sociales existentes, tal como sostiene Speranza (2019): 
En lo que se refiere específicamente a la situación de la educación superior, algunos trabajos (Espinoza, 2013) muestran que la relación entre la distribución del ingreso y el acceso a la universidad en América Latina presenta una desigualdad marcada. En el caso de la Argentina, el quintil de ingresos más altos registra un acceso a la universidad del 59\% y el quintil más bajo un acceso del 19\%. Como se observa, los jóvenes pertenecientes a los sectores sociales más favorecidos acceden a los estudios superiores en proporciones mayores respecto de los sectores menos favorecidos: "Ello prueba que las políticas de inclusión, si bien han generado un incremento de la matrícula en todos los sectores socioeconómicos, no han logrado superar los desequilibrios existentes en el acceso y la calidad de los estudios superiores" (Espinoza, 2013, p.260). La incorporación de sectores sociales históricamente relegados de la educación superior ha incrementado la heterogeneidad de los estudiantes, puesto que la mayor parte de los alumnos que pertenecen a familias migrantes integran los sectores menos favorecidos de la sociedad, en términos económicos. Esta nueva realidad genera un gran desafío para las instituciones de educación superior puesto que, en muchos casos, ofrecen resistencia a incorporarse a los cambios. (Speranza, 2019, p.312)

Esa resistencia a la incorporación de los cambios, observada en muchas instituciones, también se manifiesta en las decisiones respecto de las lenguas a las que se accede a través de acciones formales; en las decisiones sobre las lenguas que se estudian e investigan y su modo de abordaje en términos teóricos. Estas acciones dan cuenta de la concepción lingüística que subyace a las decisiones teóricas e institucionales en el marco de la política lingüística y educativa que cada institución desarrolla.

\section{El contexto institucional}

Tal como expresa Oliver (2007):

La lengua es el primer medio de comunicación entre los hombres y el más universal. Por esta condición juega un papel particular en la construcción de las identidades, y eso en múltiples niveles. A través de la lengua se forma la memoria que sostiene los sistemas identitarios y se transmiten los contenidos narrativos que son los fundamentos de las identidades. Es la lengua la que estructura, a través de sus palabras, las representaciones y los estereotipos que van a vehicular las identidades. Ella constituye el primer medio, en la historia de los individuos como en la de los grupos, de distinguir el nosotros (aquellos que comprendo, que hablan la misma lengua) de los otros (aquellos que no hablan la misma lengua y que por lo tanto son diferentes). (p.77)

La misma agenda política a la que nos referimos determina las lenguas que mayoritariamente ocuparán espacios de poder y reproducción de conocimiento, "la hegemonía de unos sistemas semióticos sobre otros" (Del Valle, 2017, p.23). Una agenda política que reduce la oferta lingüística excluye subjetividades que muchas veces aprenden las lenguas nacionales como segunda lengua y no pueden expresarse de manera satisfactoria en los géneros textuales académicos con las exigencias subyacentes. En los 
espacios académicos se privilegian las lenguas nacionales, que son también coloniales. Esta práctica marginaliza las lenguas originarias con toda su sustancia semántica, su cosmovisión y sus paisajes semióticos.

También la reflexión sobre las distintas variedades de la lengua nacional que pueblan las aulas queda, en general, fuera del sistema educativo. Sin embargo, algunos espacios universitarios han dado muestras de compromiso en esos sentidos.

\section{Una perspectiva de la teoría lingüística}

\section{El estudio de las variedades lingüísticas en contacto}

Nos referiremos, a continuación, a algunos de esos gestos incluyentes que se manifiestan en el espacio que habito como docente: la Facultad de Humanidades y Ciencias de la Educación (FAHCE) de la Universidad Nacional de la Plata (UNLP).

En efecto, los proyectos desarrollados en el área de la Lingüística arrojan luz sobre la variación del español rioplatense en contacto con otras culturas, con hablantes de quechua y guaraní, por ejemplo, y han hallado sesgos identitarios en la selección de las formas de uso de los pronombres (Martínez, 2018, 2020) en la variación verbal (Speranza, 2014; Alvarez Garriga, 2012), en el empleo de los posesivos (Risco, 2012), en el uso de las preposiciones y en la estructura textual (Bravo de Laguna, 2017). Estudios cualitativos y cuantitativos, que se nutren de corpus genuinos, revelan que los sistemas que subyacen a las variedades lingüísticas siempre aportan una información que el hablante ofrece a la comunicación y, especialmente, un perfilamiento sobre la escena representada.

Cuando ese aporte tiene que ver con rasgos culturales de la comunidad, las explicaciones se sustentan con evidencia independiente. Al decir de Martínez:

Estos análisis se hallan exentos de circularidad, fantasma que acucia a todo intento de explicación científica. Para cada interpretación cultural de los usos lingüísticos hemos aportado explicación no lingüística. En efecto, hemos presentado, en cada caso, evidencia cultural independiente. (Martínez, 2015, p.207)

Creemos que estudios como los mencionados ofrecen la posibilidad de una mirada menos estigmatizada sobre los eventos lingüísticos, porque dan lugar a una nueva comprensión sobre la conceptualización de los hechos. A este respecto, contamos con textos que dan cuenta de estas diferencias (Martínez, 2015, 2015a; Risco, 2018; Speranza, 2014) y en los cuales se entrelazan teoría y prácticas pedagógicas desde una mirada intercultural. Por otra parte, son numerosas las presentaciones en congresos desde esta perspectiva que es la adoptada, además, en los contenidos correspondientes al programa de la Cátedra de Lingüística. Si a esta preocupación por los estudios de variación, en situaciones de contacto lingüístico, sumamos la existencia del Corpus de migrantes en la Argentina 
(CORdEMIA), basado en entrevistas a bolivianos, paraguayos y peruanos residentes en La Plata y en zonas aledañas, el ambiente de prácticas interculturales se vislumbra promisorio.

\section{El concepto translanguaging en la práctica pedagógica}

Si bien, tradicionalmente, los estudios sobre el contacto de lenguas han puesto el énfasis en los sistemas lingüísticos más que en los hablantes, en la actualidad, se privilegia la idea de que el contacto se produce entre hablantes y no entre lenguas.

En efecto, aunque la perspectiva sociolingüística había considerado fundamental, desde sus comienzos, a los hablantes y a la interacción verbal para describir el fenómeno de los eventos lingüísticos, en la investigación con hablantes bilingües, se pone de manifiesto la separación que se hace entre las lenguas y sus variedades, hecho comúnmente utilizado como hipótesis que justifica "maneras de hablar".

Actualmente, el concepto translanguaging propone una diferencia fundamental en el umbral de los principios. En efecto, Otheguy, García y Reid (2015) consideran que:

Languages are not true linguistic entities because their boundaries are established on nonlinguistic grounds. Rather, they are groupings of idiolects of people with shared social, political or ethnic identities that, once so grouped, are described using linguistic terms that tend to give the mistaken impression that the grouping was based on linguistic grounds in the first place. (p.291)

En consonancia con esta aseveración, los autores ofrecen la siguiente definición de translanguaging:

The deployment of a speaker's full linguistic repertoire without regard for watchful adherence to the socially and politically defined boundaries of named (and usually national and state) languages. (Otheguy, García y Reid, 2015, p.281)

Es decir, el punto de partida de los análisis lingüísticos debería hacerse desde el repertorio del hablante. Sin embargo, de acuerdo con los autores, en general, los estudiosos de la teoría gramatical han prestado poca atención a este concepto, ante la ausencia de interés por el impacto que producen las teorías lingüísticas en la vida de los hablantes, especialmente en las minorías.

Ciertamente, si partimos del repertorio del hablante, nos enfrentamos con una posibilidad novedosa de encarar las experiencias lingüísticas. Se propone una mirada menos estigmatizada y estigmatizadora, a partir de la concepción de una gramática siempre emergente. Esta perspectiva da lugar a una visión más acogedora frente a la realidad lingüística. 
Continúan expresando los autores:

As idiolect is for us a person's own unique, personal language, the person's mental grammar that emerges in interaction with other speakers and enable the person's use of language. An idiolect is language in sense above, language viewed from the internal perspective of the individual, language seen separately from the external perspective of the society that categorizes and classifies named national languages. (Otheguy, García y Reid, 2015, p.289)

Desde la perspectiva interna del sujeto, el límite de una lengua será dado, en la interacción verbal, por el conocimiento compartido entre pares, definido por el espacio de enunciación. El sujeto en comunicación pone en juego el léxico y rasgos estructurales para un determinado fin. La selección de formas lingüísticas, desde este punto de partida, no puede ser validada solamente por estructuras que han sido determinadas por una gramática cristalizada, pues la habilidad humana excede límites que inventan mecanismos para imponer subjetividades. La lengua es el recurso que nos permite actuar y modificar nuestra realidad. Otros autores acuerdan con esta perspectiva:

Bits and pieces of words and grammatical structures from diverse languages work together for these participants because these communicative resources find coherence in terms of the spatial ecology, not necessarily in terms of the grammatical structure. Since activity is the frame of reference, not the bounded grammatical structure, it is possible to understand how diverse languages in the spatial ecology facilitate communication. (Canagarajah, 2017, p.6)

En conclusión, creemos que el impacto de este concepto será de especial relevancia para las prácticas educativas de los estudiantes bilingües, así como para la revitalización de las lenguas en comunidades minorizadas.

\section{La creación del Profesorado en portugués}

Junto a la búsqueda de profesores de lengua portuguesa en la Argentina, surge en 2014, en la FAHCE, el Profesorado en portugués. Una carrera de grado que atiende a la demanda y además ofrece un lugar importante en el quehacer académico para la lengua portuguesa -y su variación- dentro de la Universidad: no solamente forma profesores y profesoras sino también promueve la participación en la construcción del pensamiento científico. Las investigaciones sobre lengua portuguesa, así como la práctica misma de la lengua, se manifiestan en congresos y jornadas académicas y permiten mostrar los saberes y plantear aportes a las decisiones institucionales.

A partir de esas experiencias, me encuentro elaborando una tesis de doctorado que se inscribe en esos marcos teóricos relacionados con la variación lingüística y la perspectiva que propone el concepto de translanguaging. La construcción de la tesis nos ha llevado a pensar en la incorporación, en nuestra práctica docente, de distintas maneras de reflexionar sobre el lenguaje a la luz de las necesidades locales, en un ejercicio integrador, más conforme -creemos- a propuestas de enseñanza que contemplan los intereses reales de la comunidad educativa. 
Como integrante de los grupos de estudio y proyectos de investigación que se llevan a cabo en la Facultad, se nos hace posible estudiar la matriz de algunos cambios que se van observando en situaciones de contacto entre las lenguas portuguesa y española, a partir de las nuevas migraciones de brasileños y brasileñas en la Ciudad de Buenos Aires y La Plata, como puede verse en Lopes (2019).

Las acciones que se llevan a cabo en esta carrera nos permiten considerar que el lugar que viene ocupando la lengua portuguesa en la UNLP conforma una propuesta integradora como las previstas en Arnoux (2010).

\section{CONCLUSIONES}

Desde una concepción del contacto lingüístico centrado en el hablante, hemos propuesto que la participación de más lenguas dentro del ambiente universitario puede integrar cosmovisiones y contribuir a la formación y el desarrollo de los sujetos. Para la Universidad que queremos se hace necesario posibilitar una integración abarcadora y descolonizadora que incluya las demás lenguas presentes en el espacio geográfico, que todavía están sujetas a las decisiones del Estado.

Nos hemos remitido a nuestro ámbito de trabajo, la FAHCE, que ha sido el escenario de nuestra investigación, para señalar algunos gestos que creemos motivadores de estas políticas de integración a las que nos referimos: los estudios lingüísticos orientados a la reflexión y comprensión de variedades y de situaciones de contacto de lenguas, la introducción de la perspectiva de translanguaging y la creación del Profesorado en portugués.

Hemos tenido en cuenta las reflexiones de Santos (2007):

La reforma orientada a una globalización solidaria de la universidad como bien público tiene que partir de la solidaridad y de la cooperación al interior de la red nacional de universidades; esta red nacional debe estar desde el comienzo transnacionalizada, es decir, debe integrar universidades extranjeras interesadas en formas de trans-nacionalización no mercantil. Obviamente que esas relaciones -llamadas hoy "relaciones internacionales"- ya existen; solo que deben ser intensificadas hasta el punto que sean tan constitutivas de la red, que dejen de ser consideradas externas o apéndices. (Santos 2007, p.81)

$\mathrm{Y}$, en ese contexto, consideramos que para que sea posible el ejercicio de una nueva ciudadanía que contemple la conformación de identidades colectivas se hace necesario un doble movimiento: que la comunidad -con todo su bagaje-intervenga en la Universidad y que la Universidad intervenga en la comunidad en un juego de enseñanza y aprendizaje de saberes, cosmovisiones que se fortalezcan y permitan la participación de todos, en el desarrollo local y luego en el internacional. 


\section{REFERENCIAS BIBLIOGRÁFICAS}

Álvarez Garriga, D. (2012). Estudio sobre la variación perfecto simple y perfecto compuesto en los discursos presidenciales de Evo Morales: marcas del contacto lingüístico. En A. Martínez y A. Speranza (Eds.), Etnopragmática. Cuadernos de la ALFAL, 4 (pp.30-44). https://mundoalfal.org/?q=es/content/cuadernos-de-la-alfal-n\%C2\%BA4

Arnoux, E. Narvaja de. (2010). Representaciones sociolingüísticas y construcción de identidades colectivas en el Mercosur. En M. Celada, A. Fanjul y S. Nothstein (Eds.), Lenguas en un espacio de integración: acontecimientos, acciones, representaciones (pp. 17-38). Biblos.

Arnoux, E. Narvaja de. (2019). Embates de la "nueva economía". Reflexiones glotopolíticas acerca de la enseñanza de lenguas otras. Intersecciones. Revista da APEESP, 4, 11-36.

Bravo de Laguna, G. (2017). Variación morfosintáctica: cómo introducen el discurso referido los migrantes bolivianos en la ciudad de la Plata [ponencia]. XVIII Congreso de la ALFAL. Universidad Nacional de Colombia, Bogotá.

Bourdieu, P. (2008). ¿Qué significa hablar? Akal.

Canagarajah, S. (2018). Translingual practice as spatial repertoires: Expanding the paradigm beyond structuralist orientations. Applied Linguistics, 39(1), 31-54.

Del Valle, J. (2017). La perspectiva glotopolítica y la normatividad. Anuario de glotopolítica, 1, 17-39.

Gentili, P. (2008). Una vergüenza menos, una libertad más. En E. Sader, P. Gentili, H. Aboites, La reforma universitaria: desafíos y perspectivas noventa años después (pp. 36-50). CLACSO.

Lopes, B. da Silva Santana. (2019). Translanguaging como prática discursiva de brasileiros e brasileiras na Argentina. Cuadernos de la ALFAL, 11(2), 173-185.

Martínez, A. (2015). Las escuelas del Mercosur: la trama de las gramáticas y el concepto de identidades dinámicas. En E. Narvaja de Arnoux y R. Bein (Eds), Política lingüística y enseñanza de lenguas (pp. 109-134). Biblos.

Martínez, A. (2015a). ¿Cómo afecta la cultura a la gramática?: El caso de los clíticos en el español americano. Círculo de lingüística aplicada a la comunicación, 61, 186-210.

Martínez, A. (2018). El proyecto etnopragmático en el estudio del contacto de lenguas. En A. Regúnaga, S. Spinelli y M. Orden, (Comp.), Libro de Actas del IV Encuentro de Lenguas Indígenas Americanas (ELIA) (pp. 465-484). Universidad Nacional de La Pampa. 
Martínez, A. (2020). Variedades lingüísticas del español. Cómo la cultura se inscribe en la sintaxis. Revista Studi Italiani de Lingüística Teorica e Applicata, XLIX (1), 11-26.

Olliver, B. (2007). Identitè el identification. Sens mots et techniques. Lavoisier.

Otheguy, R.; García, O. \& Reid, W. (2015). Clarifying translanguaging and deconstructing named languages. A perspective from linguistics. Applied Linguistics Review, 6(3), 281-307.

Risco, R. (2012). El doble posesivo en el español andino: un enfoque etno-pragmático. En A. Martínez y A. Speranza (Eds.), Etnopragmática. Cuadernos de la ALFAL, 4, (97111). www.mundoalfal.org.

Risco, R. (Coord.). (2018). Estudios de variación y contacto lingüístico en el español peruano. Universidad Nacional de La Plata. Facultad de Humanidades y Ciencias de la Educación. http://libros.fahce.unlp.edu.ar/index.php/libros/catalog/book/108

Santos, B. Sousa dos. (2007). La universidad en el siglo XXI. Para una reforma democrática y emancipadora de la universidad. Umbrales: Revista del Postgrado en Ciencias del Desarrollo, 15, 13-70.

Speranza, A. (2014). La evidencialidad en el español americano. La expresión lingüística de la perspectiva del hablante. Iberoamericana.

Speranza, A. (2019). La urdimbre del habla urbana. El caso de la educación superior. Forma y Función, 32 (2) 299-314.

\footnotetext{
${ }^{1}$ Es doctoranda en Letras con énfasis en Lingüística por la Universidad Nacional de La Plata (UNLP). Integrante del proyecto de investigación Fronteras Teóricas III, con lugar de trabajo en el Centro de Estudios e Investigaciones Lingüísticas (CEIL). Se desempeña como profesora adjunta en la cátedra Análisis Comparativo de las Lenguas con funciones en Lingüística Aplicada, ambas del Profesorado en Portugués de la UNLP. Se dedica a la variación ocasionada por el contacto lingüístico bajo la perspectiva translingüística.

${ }^{2}$ Dadas las pautas de edición de la Revista, utilizo estrategias de lenguaje inclusivo que conservan la normativa.
} 